\title{
Review Article \\ Early Gastric Cancer: Current Advances of Endoscopic Diagnosis and Treatment
}

\author{
Linlin Zhu, ${ }^{1}$ Jinyu Qin, ${ }^{1}$ Jin Wang, ${ }^{1}$ Tianjiao Guo, ${ }^{1}$ Zijing Wang, ${ }^{2}$ and Jinlin Yang ${ }^{1}$ \\ ${ }^{1}$ Department of Gastroenterology, West China Hospital of Sichuan University, No. 37 Guo Xue Xiang, Chengdu 610041, China \\ ${ }^{2}$ Department of Gastroenterology, Kanazawa University Hospital, Kanazawa, Ishikawa 920-8641, Japan \\ Correspondence should be addressed to Jinlin Yang; mouse-577@163.com
}

Received 12 July 2015; Revised 6 November 2015; Accepted 10 November 2015

Academic Editor: Robert Odze

Copyright (c) 2016 Linlin Zhu et al. This is an open access article distributed under the Creative Commons Attribution License, which permits unrestricted use, distribution, and reproduction in any medium, provided the original work is properly cited.

\begin{abstract}
Endoscopy is a major method for early gastric cancer screening because of its high detection rate, but its diagnostic accuracy depends heavily on the availability of endoscopic instruments. Many novel endoscopic techniques have been shown to increase the diagnostic yield of early gastric cancer. With the improved detection rate of EGC, the endoscopic treatment has become widespread due to advances in the instruments available and endoscopist's experience. The aim of this review is to summarize frequently-used endoscopic diagnosis and treatment in early gastric cancer (EGC).
\end{abstract}

\section{Introduction}

Gastric cancer is the fourth most common cancer worldwide and the second leading cause of cancer death [1]. With the raised public awareness on early diagnosis and treatment of cancer as well as the development of endoscopic imaging and image enhanced techniques, such as magnification narrow-band imaging, chromoendoscopy, and confocal laser endomicroscopy, the proportion of early gastric cancer (EGC) at diagnosis is increasing. Early detection is essential for treatment. It has been shown that the prognosis of EGC is excellent with a 5-year survival rate of over 90\% [2-4].

According to the PARIS classification of superficial neoplastic lesions in the digestive tract [5], type 0 is divided into three categories corresponding to protruding lesions $(0-\mathrm{I})$, nonprotruding and nonexcavated lesions $(0-\mathrm{II})$, and excavated lesions (0-III). Type 0 -I is subdivided into pedunculated (0-Ip) and sessile (0-Is) lesions. Type 0-II is divided into three subtypes, a, b, and c, corresponding to slightly elevated, flat, and depressed lesions. Type 0-III is all ulcer. Mixed patterns with elevation and depression also occur and are classified into two groups: in 0-IIc+0-IIc lesions, most of the surface is depressed; elevation is present in a segment of the lesion at the periphery; in 0 -IIa+0-IIc lesions, there is a central depression in a globally elevated lesion. The combined patterns of excavation and depression are termed 0-III+IIc or 0-IIc+III, depending on the respective surface of the ulcer and of the depressed area.

The highest risk of invasion is seen in protruding (0-I) or depressed (0-IIc) lesions. The relation between the depth of invasion into the submucosa and lymph node metastases was analyzed in 1091 cases at the National Cancer Center Hospital in Tokyo [5]. When the invasion was less than the cut-off limit of $500 \mu \mathrm{m}$, the proportion was only $6 \%$; beyond this limit it increased to $21 \%$ [5].

Techniques of endoscopic treatment for early gastric cancer include endoscopic mucosal resection (EMR) and endoscopic submucosal dissection (ESD). The results of EMR in treating EGC are comparable to that of surgery in selected cases. ESD has been shown to increase en bloc resection of lesions regardless of their size, location, or presence of scarring $[2,6,7]$. As a class of minimally invasive endoscopic techniques, ESD is characterized by fewer traumas and complications and better therapeutic effects.

\section{Endoscopic Diagnosis}

China guidelines "China Consensus for diagnosis, treatment and endoscopic screening of early gastric cancer, 2014" recommended the high risk populations: (1) >40 years; (2) Helicobacter pylori infection; (3) previous precancerous diseases such as chronic atrophic gastritis, gastric polyps, 
gastric ulcer, and pernicious anemia; and (4) other high risk factors such as alcohol, smoking, high salt, preserved food [12].

White light endoscopy (WLE) can only detect obvious morphological changes of neoplastic lesions, such as changes in their color (redness), surface contour, or dynamic response to air/gas insufflations [8]. EGC can be divided into 3 types: elevated, superficial, and depressed. The superficial type is further subdivided into superficial elevated, superficial flat, and superficial depressed. It is difficult to find superficial flat lesions in the conventional WLE, which often cause misdiagnosis and missed diagnosis. The most common lesions of EGC were usually manifested by erythema and erosion.

Endoscopic ultrasonography (EUS) is used to check the exact structure of each layer of the gastric wall. It could be used to evaluate the infiltration depth of EGC or judge the lymph node metastasis, providing evidence for therapeutic choice. Fluorescence endoscopy can identify precancerous and some hidden lesions on the basis of fluorescence. However, the high demand of the equipment results in higher inspection cost, making its routine clinical use impossible or limited.

This review aims to summarize frequently used endoscopic diagnosis and treatment methods in EGC, such as magnifying endoscopy with narrow-band imaging (MENBI), confocal laser endomicroscopy (CLE), EMR, and ESD.

2.1. Magnifying Endoscopy with Narrow-Band Imaging. Magnifying endoscopy with narrow-band imaging (ME-NBI) is a recently developed technique, which is the combination of magnification endoscopy and narrow-band imaging. It is widely used in the detection of EGC based on the basic microanatomical findings of the microvascular (MV) pattern and microsurface (MS) structures of the superficial mucosa. The gastric mucosa is composed of glandular epithelium,which is different from the normal glands. There were many different classification systems to describe the correlation between the microanatomy and actual images visualized using ME-NBI.

The MV and MS patterns were reported by Yao and others $[8,13-15]$, where three microvascular/microsurface patterns were described: regular, irregular, and absent (Table 1). According to these MS/MV patterns, one could differentiate gastric low-grade adenoma from EGC or determine the lateral extent and the tumor invasion depth of early gastric cancer for curative endoscopic resection [11]. The criteria for diagnosing gastric cancer depend on the presence of irregular MS/MV pattern with a demarcation line [8]. It is worth mentioning that $97 \%$ of EGC fits the above criteria [16]. Kaise et al. examined the significance of various MS and MV changes, such as the disappearance of the MS pattern, a change in vessel caliber, and heterogeneity in appearance. These criteria determined the diagnosis of cancer with a sensitivity of $69.1 \%$ and a specificity of $85.3 \%$ [10]. To differentiate between adenocarcinoma and undifferentiated adenocarcinoma, Nakayoshi et al. classified the MV patterns of superficial gastric cancers into three groups: fine network pattern, corkscrew pattern, and unclassified pattern [11, 17].
Yokoyama et al. found that the intralobular loop (ILL) pattern and the presence of fine network or corkscrew vessels on MV pattern could be related to histological subtype, but it is not clear whether these criteria might be universally applied $[9,13]$.

Kobara et al. and Kikuchi et al. reported that ME-NBI can be used to determine the invasion depth in gastric cancer $[7,18]$. Kobara et al. described three indicators: nonstructure, scattery, and multicaliber vessels. They suggested that the presence of all three indicators or the presence of two or more indicators with ME-NBI should be considered as diagnostic criteria of submucosa 2 (SM2) for depressed gastric cancer [18]. Kikuchi et al. found that the presence of dilated vessels (vessels with a diameter 3 times larger than that of the irregular microvessels that are frequently observed in the lesions) should be used to predict submucosal carcinoma; the specificity and negative predictive value (NPV) were close to $90 \%$, but the sensitivity of dilated vessels was only $37.5 \%$ [7]. Kiyotoki et al. compared ME-NBI and indigo carmine chromoendoscopy (ICC) to evaluate the tumor margins and found that the accurate rate which making of ME-NBI was significantly higher than that of the ICC (97.4\% versus $77.8 \%$; $P=0.009$ ) [19].

2.2. Confocal Laser Endomicroscopy. Confocal laser endomicroscopy (CLE) is a newly developed endoscopic imaging technology, which produces 1000 -fold magnification crosssectional images of the GI surface and subsurface tissue. It has the ability to provide a direct histological observation of the in vivo tissue without the need for biopsy and to differentiate malignant from benign lesions in real time at the cellular level [20]. Gastric pit is the smallest unit of the gastric mucosal surface and the smallest structural unit in the confocal images. It presents as different images in different disease states [21]. According to the classification of gastric pit patterns by Zhang et al. [20], the gastric pit patterns were divided into 7 types. Normal mucosa with fundic glands mainly contains round pits (type A). Type $G$ is usually found on gastric cancer under CLE images, whose sensitivity and specificity for gastric cancer were $90 \%$ and $99.4 \%$, respectively. Type G1 is defined as the loss of normal gastric pits accompanied by the appearance of diffusely atypical cells, such as signet ring cell cancer and low differentiated tubular adenocarcinoma. Type G2 is manifested by the loss of normal pits with the appearance of atypical glands, mainly in well differentiated tubular adenocarcinoma [20, 22].

CLE has great advantage on microvascular imaging, since blood vessels of normal and cancerous mucosa have different characteristics under CLE [23]. At present, the cellular changes and the tissue and vascular structure of CLE in the diagnosis of gastric mucosal lesions were based on sodium fluorescein staining. A prospective study involving 1786 patients was carried out by $\mathrm{Li}$ et al. to evaluate the validity and reliability of the CLE in the identification of gastric superficial cancerous lesions. They found that CLE diagnosis for early gastric cancers had high sensitivity (88.1\%) and specificity (98.6\%) [24]. Kakeji et al. examined normal and 27 gastric cancer tissues ex vivo. Compared to histology outcomes, CLE had a high diagnostic accuracy of $96.4 \%$, 
TABLE 1: Microvascular/microsurface patterns.

\begin{tabular}{ll}
\hline Study & Microvascular pattern
\end{tabular}

Regular microvascular pattern

Closed- or open-looped with a uniform shape

Homogeneous morphology

Symmetrical distribution

Regular arrangement

Different shapes: closed-looped

Yao, $2013[8]$

Irregular microvascular pattern

(polygonal), open-looped, tortuous,

branched, bizarrely shaped

Heterogeneous morphology

Asymmetrical distribution

Irregular arrangement

Subepithelial microvascular pattern is obscured

Absent microvascular pattern

Presence of a white opaque substance (WOS)

Microsurface pattern

Regular microsurface pattern

Shape of marginal crypt epithelium: uniform linear/curved/oval/circular structure

Homogeneous morphology

Symmetrical distribution

Regular arrangement

Regular WOS

Yao, 2013 [8]

Shape of marginal crypt epithelium: irregular linear/curved/oval/circular structure

Irregular microsurface pattern

Heterogeneous morphology

Asymmetrical distribution

Irregular arrangement

Irregular WOS

Absent microsurface pattern

No marginal crypt epithelial structure No WOS are visible

Fine network pattern

Corkscrew pattern

Intralobular loop pattern-1

Intralobular loop pattern-2

Yokoyama et al., 2010 [9] Abnormal microvascular irregular superficial glandular pattern

Fine mucosal structural features

Absence

Abrupt caliber alteration

Denseness

Heterogeneity in shape

Micrification

Tortuousness

Heterogeneity

A fine network pattern

A corkscrew pattern

An unclassified pattern

and the sensitivity and specificity were $92.6 \%$ and $100 \%$, respectively [25]. Kitabatake et al. obtained in vivo CLE images from normal mucosa and cancerous lesions in 27 patients with EGC and demonstrated sensitivity of $81.8 \%$, specificity of $97.6 \%$, and accuracy of $94.2 \%$ [26].

\subsection{Endoscopic Treatment. The new NCCN guideline [27]} recommended that EMR or ESD of early stage gastric cancer can be considered as adequate therapy when the lesion is $\leq 2 \mathrm{~cm}$ in diameter; it is shown on histopathology that it is well or moderately well differentiated, does not penetrate beyond the superficial submucosa, does not exhibit lymphovascular invasion, and has clear lateral and deep margins. But the guidelines did not specify when is EMR and when is ESD indicated.

The following are risk factors in case of endoscopic treatment: (1) failure of the lesion to lift after injection of saline into the submucosa (the nonlifting sign), (2) early gastric cancer with lymph node metastasis, (3) cancer invasion of the muscularis propria, and (4) severe coagulation 
dysfunction. Age is not a risk factor, except for severe organ failure. Anticoagulation should be stopped 5-7 days before the procedure [28].

2.4. Endoscopic Mucosal Resection (EMR). Endoscopic mucosal resection was first introduced for endoscopic therapy in 1984 by using the strip biopsy method (twochannel method) $[29,30]$. The operation process included submucosal injection under the lesion, snaring, and removing the lesion. This injection-snaring method is simple and convenient. However, it is difficult to trap flat type lesions. Furthermore, the steel wire slips easily, which may lead to incomplete resection and local recurrence [31]. EMR after circumferential precutting was described by Hirao et al. in 1988 [30, 32]. The injection-precutting-snaring (EMR-P) technique refers to submucosal injection of hypertonic saline mixed with diluted epinephrine, cut around the lesion with a needle knife, and removal of the lesion by a snare. In summary, the injection-snaring and injection-precuttingsnaring techniques are noninhalation methods of EMR. The inhalation techniques of EMR include EMR with a cap (EMR-C) and EMR with ligation (EMR-L). EMR-C was developed in 1992 [33]. This technique can safely remove intramucosal cancers $2 \mathrm{~cm}$ or less in diameter by using transparent plastic cap that is connected to the tip of an endoscope. The procedure includes submucosal injection, suction into the cap, and snare and resection [2, 34]. The operation process of EMR with ligation (EMR-L) is similar to that of EMR-C. The equipment of EMR-L is a standard variceal ligation device. After sucking the lesion into the cap, lodged band is deployed underneath the lesion, and then the banded lesion is snared and removed $[2,35]$. The traditional EMR methods (mentioned above) could not remove huge flat lesions for more than $2 \mathrm{~cm}$ one-time. This kind of lesions could be resection in several parts by endoscopy piecemeal mucosal resection (EPMR). However, it is difficult to splice the resected samples in vitro and evaluate the efficacy of radical resection after EPMR.

The Japan Gastroenterological Endoscopy Society (JGES) in collaboration with the Japanese Gastric Cancer Association (JGCA) has created guidelines for ESD and EMR for the treatment of EGC in 2015 [36]. The guidelines recommend that endoscopic resection should be carried out when the likelihood of lymph node metastasis is extremely low and lesion size and site are amenable to resection en bloc. Endoscopic therapy (EMR or ESD) is absolutely indicated in macroscopically intramucosal (cT1a) differentiated carcinomas measuring less than $2 \mathrm{~cm}$ in diameter. The macroscopic type does not matter, but there must be no finding of ulceration (scar), that is, $\mathrm{UL}(-)$.

The outcomes of EMR showed $56 \%-75.8 \%$ en bloc rates and $66.1 \%-77.6 \%$ complete resection rates in foreign countries [37-41]. A multicenter study on EMR in the treatment of early gastric cancer from Japan found that the complete resection rate is relevant to the lesion size; the complete resection rate of lesions less than $1 \mathrm{~cm}$ was $82.4 \%$, while only $16.2 \%$ of those larger than $2 \mathrm{~cm}$ were resected completely [37]. Another Japanese literature reported that both 5-year and 10year survival rates of patients with mucosal EGC less than
$2 \mathrm{~cm}$ that were completely removed by EMR were 99\% [2, 42]. The risk of local recurrence after EMR ranged from 2\% to $35 \%$ in Japan [38]. Horiki et al. found that the local recurrence rate of early gastric cancer after piecemeal EMR was 30\% (95\% CI, 20-40\%) at both 5 and 10 years [43]. Additional surgery was performed soon after the initial EMR if a resection margin was clearly positive for cancer. Furthermore, identification of the resection margin is a problem in EPMR.

2.5. Endoscopic Submucosal Dissection (ESD). With the limitations of EMR resection of lesions, people seek to find new endoscopic techniques to remove larger tissues. Endoscopic submucosal dissection (ESD) permits en bloc resection of larger lesions [2, 44-50]. The steps of this endoscopic technique consist of marking, submucosal injection, circumferential mucosal precutting, dissection, and dealing with wound. The current indications for ESD are based on the criteria reported by Gotoda and colleagues, which include an ulcerative mucosal EGC $<3 \mathrm{~cm}$ in diameter or a submucosal invasion depth of the EGC $\leq 3 \mathrm{~cm}$ [51].

For ESD indications, according to the recent Japanese Gastric Cancer guidelines in 2015 [36] and ESMO-ESSOESTRO in 2013, in addition to EMR's indications, the ESD's indications include the following:

(1) UL(-) cTla differentiated carcinomas greater than $2 \mathrm{~cm}$ in diameter.

(2) $\mathrm{UL}(+)$ cTla differentiated carcinomas less than $3 \mathrm{~cm}$ in diameter.

(3) UL(-) cTla undifferentiated carcinomas less than $2 \mathrm{~cm}$ in diameter.

(4) The extremely low risk of lymph node metastasis and the possibility of it becoming reasonable to expand the indications when vascular infiltration (ly, v) is absent together with the above-mentioned criteria.

(5) The possibility of dealing with subsequent locally recurrent intramucosal cancers under expanded indications (evidence level V, grade of recommendation C1) if a lesion falls within the indication criteria at the initial ESD or EMR.

According to the literature, the en bloc resection rate of ESD for early gastric cancer was 94.9\%-97.7\% and 5-year survival rate was $83.1 \%-97.1 \%$ [52-57]. A multicenter retrospective study comparing EMR and ESD with resection in early gastric cancer reported that the one-piece resection rate with ESD was significantly higher than that with EMR $(92.7 \%$ versus 56\%) [41]. The incidence of perforation was 3.6\% with ESD and 1.2\% with EMR, although the complications were managed endoscopically. The 3-year cumulative residualfree/recurrence-free rate of ESD was 97.6\%. In another study stated by Toyonaga et al. involving 1136 patients with gastric cancer, the en bloc resection was $97.1 \%$, bleeding and perforation rates were $3.6 \%$ and $1.8 \%$, and the 3 -year and 5year survival rates were $91.7 \%$ and $88.1 \%$ [58].

The mainly postoperative complications of ESD included bleeding, perforation, and stenosis. Acute intraoperative bleeding rate was $3.1-15.6 \%$ and the rate of delayed bleeding 
was $3.1 \% \sim 15.6 \%$ [59]. Bleeding may be associated with the size of lesions more than $4 \mathrm{~cm}$ or the thick submucosal blood vessels located in the upper two-thirds of the stomach [60, 61]. Perforation rate of ESD was $1.2 \%-4.1 \%$; the risk factor of perforation is that the lesions more than $2 \mathrm{~cm}$ [59]. According to the study carried out by Coda et al., the stenosis rate after gastric body ESD was $17 \%$ (7/41), while $7 \%$ (8/115) pylorus stenosis occurred after ESD [62]. The study identified the following as the risk factors of stenosis, the surface of the mucosal circumferential defect that achieved to more than $3 / 4$, or the length of the removal mucosal more than $5 \mathrm{~cm}$.

As the kind of knives and the skill level of the operators were different, the proper number of cases required to gain adequate experience for ESD remains debatable [63-65]. Several studies suggested that experience comprising at least 30 cases overall and 30 cases in the lower third of the stomach [66] are needed for a beginner/trainee. Others suggested that a beginner could begin with lesions in the lower part of the stomach after 30 supervised ESD procedures [63]. A recent study suggested that experience with 30 procedures was not enough to complete all gastric ESDs without expert help for novice operators [67].

Procedure time was suggested as a marker of proficiency of the ESD. Bleeding control skills during submucosal dissection are a key feature of ESD $[65,67]$. Hong et al. investigate the learning curve of ESD of gastric neoplasms by assessing the following parameters: en bloc resection rate, complete resection rate, duration and speed of procedure time, and related complications [68]. They found that the procedure time was significantly longer for lesions in the upper third of the stomach compared with lesions located in the middle third and lower third of the stomach $(P=0.01$ and 0.01$)$. Specimen size over $1501 \mathrm{~mm}^{2}$ was correlated with a longer procedure time in comparison with specimen size under 500 , 501-1000, and 1001-1500 $\mathrm{mm}^{2}(P=0.02, P<0.01$, and $P<0.01)$. En bloc resection rates and complete resection rates were not significantly related to the sequences of ESD procedures. The frequency of bleeding and perforation was not related to the sequence of treatment cases. This study suggested that novice operators begin with cases involving easy sites and small areas, which could lead to a higher complete resection rate compared with expert operators [68].

It is difficult to remove large lesions for ESD, as the lifting effect of the submucosal injection is less obvious after the circumferential incision than before it; the endoscopic view is obstructed or reduced when the resection reaches the central portion due to the confined space and contraction of the resected mucosa [69]. Local resection can be less accurate at evaluating the exact status of lymphovascular invasion and lymph node metastasis than at surgery [70]. It causes additional gastrectomy if the depth of invasion is deeper than the SM2 layer.

Endoscopic submucosal tunnel dissect (ESTD) ion was first introduced by Linghu et al. as a new strategy for rapid resection of large esophageal neoplasms [69]. A tunnel was established between the mucosa and the muscularis propria; then the lesions were resected rapidly. The use of ESTD to remove EGC has limited indications such as EGC with severe fibrosis due to previous ESD or severe ulceration and achievement of a sufficient resection margin because of submucosal invasion [71]. Choi et al. [71] reported two cases of ulcerative early gastric cancer with submucosal fibrosis that were treated by endoscopic submucosal tunnel dissection. It cannot be denied that ESTD has some advantages. For example, bleeding is easier to control due to the clearly exposed blood vessels, and the operation time is shortened. More research is required to assess the safety and effectiveness of the ESTD for resection of EGC.

\section{Conclusion}

In summary, with the development of endoscopic techniques, more prospective studies with high-quality designs should be performed to evaluate the diagnostic accuracy of these new endoscopic imaging techniques to add up the therapeutic outcomes, survival rates, and complication rates and to standardize the procedures and develop a learning system which is widely acceptable by endoscopists in the future.

\section{Conflict of Interests}

The authors declare that there is no conflict of interests regarding the publication of this paper.

\section{Authors' Contribution}

Linlin Zhu drafted the paper; Jinyu Qin, Jin Wang, and Tianjiao Guo performed the systematic search of literature; Zijing Wang performed the revision of the paper; Jinlin Yang designed the study and edited the paper.

\section{References}

[1] A. Jemal, F. Bray, M. M. Center, J. Ferlay, E. Ward, and D. Forman, "Global cancer statistics," CA Cancer Journal for Clinicians, vol. 61, no. 2, pp. 69-90, 2011.

[2] Y. W. Min, B.-H. Min, J. H. Lee, and J. J. Kim, "Endoscopic treatment for early gastric cancer," World Journal of Gastroenterology, vol. 20, no. 16, pp. 4566-4573, 2014.

[3] F. J. Oliveira, H. Furtado, E. Furtado, H. Batista, and L. Conceicao, "Early gastric cancer: report of 58 cases," Gastric Cancer, vol. 1, no. 1, pp. 51-56, 1998.

[4] H. Ono, H. Kondo, T. Gotoda et al., "Endoscopic mucosal resection for treatment of early gastric cancer," Gut, vol. 48, no. 2, pp. 225-229, 2001.

[5] P. W. Participants, "The Paris endoscopic classification of superficial neoplastic lesions," Gastrointestinal Endoscopy, vol. 58, no. 6, pp. S1-S22, 2003.

[6] S. Hoteya, T. Iizuka, D. Kikuchi, and N. Yahagi, "Benefits of endoscopic submucosal dissection according to size and location of gastric neoplasm, compared with conventional mucosal resection," Journal of Gastroenterology and Hepatology, vol. 24, no. 6, pp. 1102-1106, 2009.

[7] D. Kikuchi, T. Iizuka, S. Hoteya et al., "Usefulness of magnifying endoscopy with narrow-band imaging for determining tumor invasion depth in early gastric cancer," Gastroenterology Research and Practice, vol. 2013, Article ID 217695, 5 pages, 2013.

[8] K. Yao, "The endoscopic diagnosis of early gastric cancer," Annals of Gastroenterology, vol. 26, no. 1, pp. 11-22, 2013. 
[9] A. Yokoyama, H. Inoue, H. Minami et al., "Novel narrow-band imaging magnifying endoscopic classification for early gastric cancer," Digestive and Liver Disease, vol. 42, no. 10, pp. 704-708, 2010.

[10] M. Kaise, M. Kato, M. Urashima et al., "Magnifying endoscopy combined with narrow-band imaging for differential diagnosis of superficial depressed gastric lesions," Endoscopy, vol. 41, no. 4, pp. 310-315, 2009.

[11] T. Nakayoshi, H. Tajiri, K. Matsuda, M. Kaise, M. Ikegami, and H. Sasaki, "Magnifying endoscopy combined with narrow band imaging system for early gastric cancer: correlation of vascular pattern with histopathology (including video)," Endoscopy, vol. 36, no. 12, pp. 1080-1084, 2004.

[12] C.s.o.d. endoscopy, "China Consensus for diagnosis, treatment and endoscopic screening of early gastric cancer, 2014," Chinese Journal of Digestion, vol. 31, no. 7, pp. 433-447, 2014.

[13] B. Hayee, H. Inoue, H. Sato et al., "Magnification narrowband imaging for the diagnosis of early gastric cancer: a review of the Japanese literature for the Western endoscopist," Gastrointestinal Endoscopy, vol. 78, no. 3, pp. 452-461, 2013.

[14] K. Yagi, A. Nakamura, and A. Sekine, "Characteristic endoscopic and magnified endoscopic findings in the normal stomach without Helicobacter pylori infection," Journal of Gastroenterology and Hepatology, vol. 17, no. 1, pp. 39-45, 2002.

[15] K. Yao, S. Nakagawa, M. Nakagawa et al., "Gastric microvascular architecture as visualized by magnifying endoscopy: body and antral mucosa without pathologic change demonstrate two different patterns of microvascular architecture," Gastrointestinal Endoscopy, vol. 59, no. 4, pp. 596-597, 2004.

[16] K. Yao, G. K. Anagnostopoulos, and K. Ragunath, "Magnifying endoscopy for diagnosing and delineating early gastric cancer," Endoscopy, vol. 41, no. 5, pp. 462-467, 2009.

[17] J. Y. Jang, "The usefulness of magnifying endoscopy and narrow-band imaging in measuring the depth of invasion before endoscopic submucosal dissection," Clinical Endoscopy, vol. 45, no. 4, pp. 379-385, 2012.

[18] H. Kobara, H. Mori, S. Fujihara et al., "Prediction of invasion depth for submucosal differentiated gastric cancer by magnifying endoscopy with narrow-band imaging," Oncology Reports, vol. 28, no. 3, pp. 841-847, 2012.

[19] S. Kiyotoki, J. Nishikawa, M. Satake et al., "Usefulness of magnifying endoscopy with narrow-band imaging for determining gastric tumor margin," Journal of Gastroenterology and Hepatology, vol. 25, no. 10, pp. 1636-1641, 2010.

[20] J.-N. Zhang, Y.-Q. Li, Y.-A. Zhao et al., "Classification of gastric pit patterns by confocal endomicroscopy," Gastrointestinal Endoscopy, vol. 67, no. 6, pp. 843-853, 2008.

[21] J. Yang, N.-N. Fan, and Y.-S. Yang, "Application of confocal laser endomicroscopy in diagnosis of digestive tract cancer and precancerous lesions," Medical Journal of Chinese People's Liberation Army, vol. 37, no. 11, pp. 921-925, 2012.

[22] L. Y. Liu Jun, "Diagnosis of gastric cancer and precancerous changes by confocal laser endomicroscoy," Chinese Journal of Gastroenterology, vol. 19, pp. 257-260, 2014.

[23] H. Liu, Y.-Q. Li, T. Yu et al., "Confocal endomicroscopy for in vivo detection of microvascular architecture in normal and malignant lesions of upper gastrointestinal tract," Journal of Gastroenterology and Hepatology, vol. 23, no. 1, pp. 56-61, 2008.

[24] W.-B. Li, X.-L. Zuo, C.-Q. Li et al., "Diagnostic value of confocal laser endomicroscopy for gastric superficial cancerous lesions," Gut, vol. 60, no. 3, pp. 299-306, 2011.
[25] Y. Kakeji, S. Yamaguchi, D. Yoshida et al., "Development and assessment of morphologic criteria for diagnosing gastric cancer using confocal endomicroscopy: an ex vivo and in vivo study," Endoscopy, vol. 38, no. 9, pp. 886-890, 2006.

[26] S. Kitabatake, Y. Niwa, R. Miyahara et al., "Confocal endomicroscopy for the diagnosis of gastric cancer in vivo," Endoscopy, vol. 38, no. 11, pp. 1110-1114, 2006.

[27] NCCN, The NCCN Gastric Cancer Clinical Practice Guidelines in Oncology (Version 1.2015), National Comprehensive Cancer Network (NCCN), 2015.

[28] A. M. Veitch, T. P. Baglin, A. H. Gershlick, S. M. Harnden, R. Tighe, and S. Cairns, "Guidelines for the management of anticoagulant and antiplatelet therapy in patients undergoing endoscopic procedures," Gut, vol. 57, no. 9, pp. 1322-1329, 2008.

[29] M. Tada, M. Shimada, and F. Murakami, "Development of the strip-off biopsy," Gastroenterological Endoscopy, vol. 26, no. 6, pp. 833-839, 1984.

[30] E. Bollschweiler, F. Berlth, C. Baltin, S. Mönig, and A. H. Hölscher, "Treatment of early gastric cancer in the Western World," World Journal of Gastroenterology, vol. 20, no. 19, pp. 5672-5678, 2014.

[31] R. Soetikno, T. Kaltenbach, R. Yeh, and T. Gotoda, "Endoscopic mucosal resection for early cancers of the upper gastrointestinal tract," Journal of Clinical Oncology, vol. 23, no. 20, pp. 44904498, 2005.

[32] M. Hirao, K. Masuda, T. Asanuma et al., "Endoscopic resection of early gastric cancer and other tumors with local injection of hypertonic saline-epinephrine," Gastrointestinal Endoscopy, vol. 34, no. 3, pp. 264-269, 1988.

[33] H. Inoue, K. Takeshita, H. Hori, Y. Muraoka, H. Yoneshima, and M. Endo, "Endoscopic mucosal resection with a cap-fitted panendoscope for esophagus, stomach, and colon mucosal lesions," Gastrointestinal Endoscopy, vol. 39, no. 1, pp. 58-62, 1993.

[34] K. Kume, M. Yamasaki, K. Kubo et al., "EMR of upper GI lesions when using a novel soft, irrigation, prelooped hood," Gastrointestinal Endoscopy, vol. 60, no. 1, pp. 124-128, 2004.

[35] Y. Suzuki, H. Hiraishi, K. Kanke et al., "Treatment of gastric tumors by endoscopic mucosal resection with a ligating device," Gastrointestinal Endoscopy, vol. 49, no. 2, pp. 192-199, 1999.

[36] H. Ono, K. Yao, M. Fujishiro et al., "Guidelines for endoscopic submucosal dissection and endoscopic mucosal resection for early gastric cancer," Digestive Endoscopy, vol. 1, no. 10, pp. 1-13, 2015.

[37] K. Ida, S. Nakazawa, J. Yoshino et al., "Multicentre collaborative prospective study of endoscopic treatment of early gastric cancer," Digestive Endoscopy, vol. 16, no. 4, pp. 295-302, 2004.

[38] T. Kojima, A. Parra-Blanco, H. Takahashi, R. Fujita, and C. J. Lightdale, "Outcome of endoscopic mucosal resection for early gastric cancer: review of the Japanese literature," Gastrointestinal Endoscopy, vol. 48, no. 5, pp. 550-555, 1998.

[39] J. C. Park, S. K. Lee, J. H. Seo et al., "Predictive factors for local recurrence after endoscopic resection for early gastric cancer: long-term clinical outcome in a single-center experience," Surgical Endoscopy, vol. 24, no. 11, pp. 2842-2849, 2010.

[40] J. J. Kim, J. H. Lee, H.-Y. Jung et al., "EMR for early gastric cancer in Korea: a multicenter retrospective study," Gastrointestinal Endoscopy, vol. 66, no. 4, pp. 693-700, 2007.

[41] I. Oda, D. Saito, M. Tada et al., "A multicenter retrospective study of endoscopic resection for early gastric cancer," Gastric Cancer, vol. 9, no. 4, pp. 262-270, 2006. 
[42] N. Uedo, H. Iishi, M. Tatsuta et al., "Longterm outcomes after endoscopic mucosal resection for early gastric cancer," Gastric Cancer, vol. 9, no. 2, pp. 88-92, 2006.

[43] N. Horiki, F. Omata, M. Uemura et al., "Risk for local recurrence of early gastric cancer treated with piecemeal endoscopic mucosal resection during a 10-year follow-up period," Surgical Endoscopy and Other Interventional Techniques, vol. 26, no. 1, pp. 72-78, 2012.

[44] S. Oka, S. Tanaka, I. Kaneko et al., "Advantage of endoscopic submucosal dissection compared with EMR for early gastric cancer," Gastrointestinal Endoscopy, vol. 64, no. 6, pp. 877-883, 2006.

[45] Y. Takeuchi, N. Uedo, H. Iishi et al., "Endoscopic submucosal dissection with insulated-tip knife for large mucosal early gastric cancer: a feasibility study (with videos)," Gastrointestinal Endoscopy, vol. 66, no. 1, pp. 186-193, 2007.

[46] H. Yamamoto and H. Kita, "Endoscopic therapy of early gastric cancer," Best Practice and Research: Clinical Gastroenterology, vol. 19, no. 6, pp. 909-926, 2005.

[47] H. Ono, "Endoscopic submucosal dissection for early gastric cancer," Chinese Journal of Digestive Diseases, vol. 6, no. 3, pp. 119-121, 2005.

[48] A. Probst, B. Pommer, D. Golger, M. Anthuber, H. Arnholdt, and H. Messmann, "Endoscopic submucosal dissection in gastric neoplasia-experience from a European center," Endoscopy, vol. 42, no. 12, pp. 1037-1044, 2010.

[49] K. B. Cho, W. J. Jeon, and J. J. Kim, "Worldwide experiences of endoscopic submucosal dissection: not just Eastern acrobatics," World Journal of Gastroenterology, vol. 17, no. 21, pp. 2611-2617, 2011.

[50] S. Nonaka, I. Oda, T. Nakaya et al., "Clinical impact of a strategy involving endoscopic submucosal dissection for early gastric cancer: determining the optimal pathway," Gastric Cancer, vol. 14, no. 1, pp. 56-62, 2011.

[51] T. Gotoda, H. Yamamoto, and R. M. Soetikno, "Endoscopic submucosal dissection of early gastric cancer," Journal of Gastroenterology, vol. 41, no. 10, pp. 929-942, 2006.

[52] T. Gotoda, "A large endoscopic resection by endoscopic submucosal dissection procedure for early gastric cancer," Clinical Gastroenterology and Hepatology, vol. 3, no. 7, supplement 1, pp. S71-S73, 2005.

[53] I.-K. Chung, J. H. Lee, S.-H. Lee et al., "Therapeutic outcomes in 1000 cases of endoscopic submucosal dissection for early gastric neoplasms: Korean ESD Study Group multicenter study," Gastrointestinal Endoscopy, vol. 69, no. 7, pp. 1228-1235, 2009.

[54] H. Isomoto, S. Shikuwa, N. Yamaguchi et al., "Endoscopic submucosal dissection for early gastric cancer: a large-scale feasibility study," Gut, vol. 58, no. 3, pp. 331-336, 2009.

[55] M. Tanaka, H. Ono, N. Hasuike, and K. Takizawa, "Endoscopic submucosal dissection of early gastric cancer," Digestion, vol. 77, no. 1, pp. 23-28, 2008.

[56] M. K. Choi, G. H. Kim, D. Y. Park et al., "Long-term outcomes of endoscopic submucosal dissection for early gastric cancer: a single-center experience," Surgical Endoscopy and Other Interventional Techniques, vol. 27, no. 11, pp. 4250-4258, 2013.

[57] T. Kosaka, M. Endo, Y. Toya et al., "Long-term outcomes of endoscopic submucosal dissection for early gastric cancer: a single-center retrospective study," Digestive Endoscopy, vol. 26, no. 2, pp. 183-191, 2014.

[58] T. Toyonaga, M. Man-I, J. E. East et al., "1,635 endoscopic submucosal dissection cases in the esophagus, stomach, and colorectum: complication rates and long-term outcomes," Surgical Endoscopy, vol. 27, no. 3, pp. 1000-1008, 2013.

[59] H. Z. W. Wu, F. Liu, and Z. S. Li, "Clinical advances on endoscopic resection for early gastric cancer and precancerous lesions," Chinese Journal of Practical Internal Medicine, vol. 34, pp. 530-538, 2014.

[60] I. Oda, T. Gotoda, H. Hamanaka et al., "Endoscopic submucosal dissection for early gastric cancer: technical feasibility, operation time and complications from a large consecutive series," Digestive Endoscopy, vol. 17, no. 1, pp. 54-58, 2005.

[61] K. Okada, Y. Yamamoto, A. Kasuga et al., "Risk factors for delayed bleeding after endoscopic submucosal dissection for gastric neoplasm," Surgical Endoscopy, vol. 25, no. 1, pp. 98-107, 2011.

[62] S. Coda, I. Oda, T. Gotoda, C. Yokoi, T. Kikuchi, and H. Ono, "Risk factors for cardiac and pyloric stenosis after endoscopic submucosal dissection, and efficacy of endoscopic balloon dilation treatment," Endoscopy, vol. 41, no. 5, pp. 421-426, 2009.

[63] N. Kakushima, M. Fujishiro, S. Kodashima, Y. Muraki, A. Tateishi, and M. Omata, "A learning curve for endoscopic submucosal dissection of gastric epithelial neoplasms," Endoscopy, vol. 38, no. 10, pp. 991-995, 2006.

[64] I.-L. Lee, C.-S. Wu, S.-Y. Tung et al., "Endoscopic submucosal dissection for early gastric cancers: experience from a new endoscopic center in Taiwan," Journal of Clinical Gastroenterology, vol. 42, no. 1, pp. 42-47, 2008.

[65] S. Yamamoto, N. Uedo, R. Ishihara et al., "Endoscopic submucosal dissection for early gastric cancer performed by supervised residents: assessment of feasibility and learning curve," Endoscopy, vol. 41, no. 11, pp. 923-928, 2009.

[66] I. Oda, T. Odagaki, H. Suzuki, S. Nonaka, and S. Yoshinaga, "Learning curve for endoscopic submucosal dissection of early gastric cancer based on trainee experience," Digestive Endoscopy, vol. 24, supplement 1, pp. 129-132, 2012.

[67] Y. Tsuji, K. Ohata, M. Sekiguchi et al., "An effective training system for endoscopic submucosal dissection of gastric neoplasm," Endoscopy, vol. 43, no. 12, pp. 1033-1038, 2011.

[68] K. H. Hong, S. J. Shin, and J. H. Kim, "Learning curve for endoscopic submucosal dissection of gastric neoplasms," European Journal of Gastroenterology and Hepatology, vol. 26, no. 9, pp. 949-954, 2014.

[69] E. Linghu, X. Feng, X. Wang, J. Meng, H. Du, and H. Wang, "Endoscopic submucosal tunnel dissection for large esophageal neoplastic lesions," Endoscopy, vol. 45, no. 1, pp. 60-62, 2013.

[70] W. Y. Cho, J. Y. Cho, I. K. Chung, J. I. Kim, J. S. Jang, and J. H. Kim, "Endoscopic submucosal dissection for early gastric cancer: quo vadis?" World Journal of Gastroenterology, vol. 17, no. 21, pp. 2623-2625, 2011.

[71] H. S. Choi, H. J. Chun, M. H. Seo et al., "Endoscopic submucosal tunnel dissection salvage technique for ulcerative early gastric cancer," World Journal of Gastroenterology, no. 27, pp. 9210-9214, 2014. 


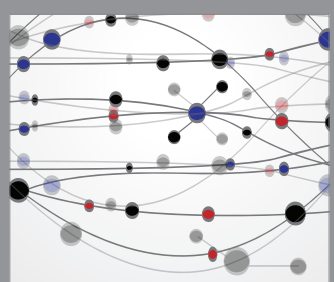

The Scientific World Journal
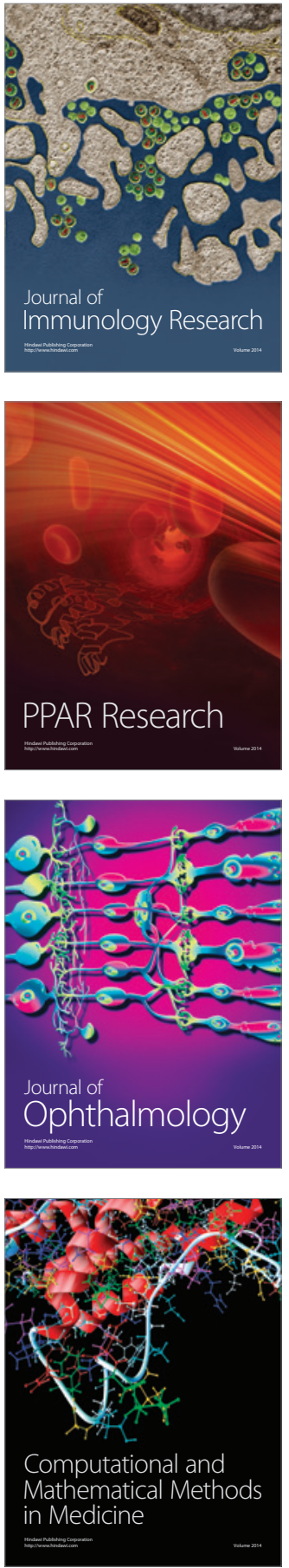

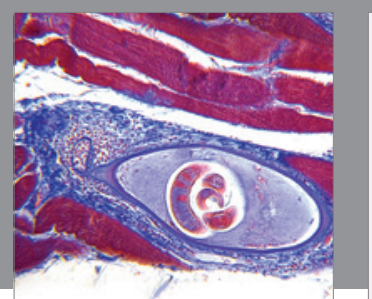

Gastroenterology Research and Practice

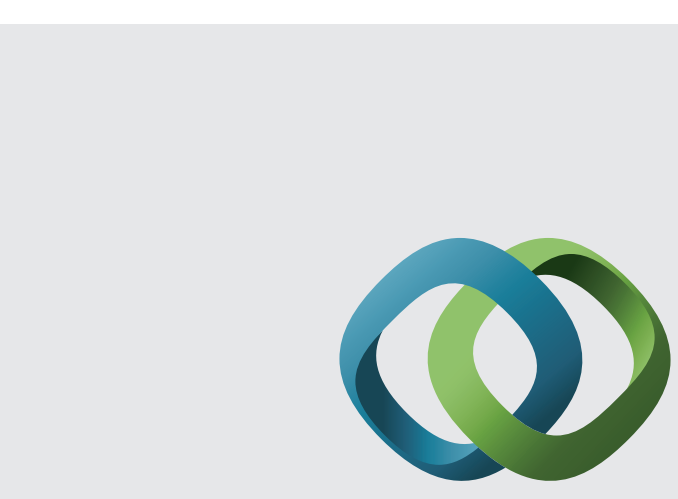

\section{Hindawi}

Submit your manuscripts at

http://www.hindawi.com
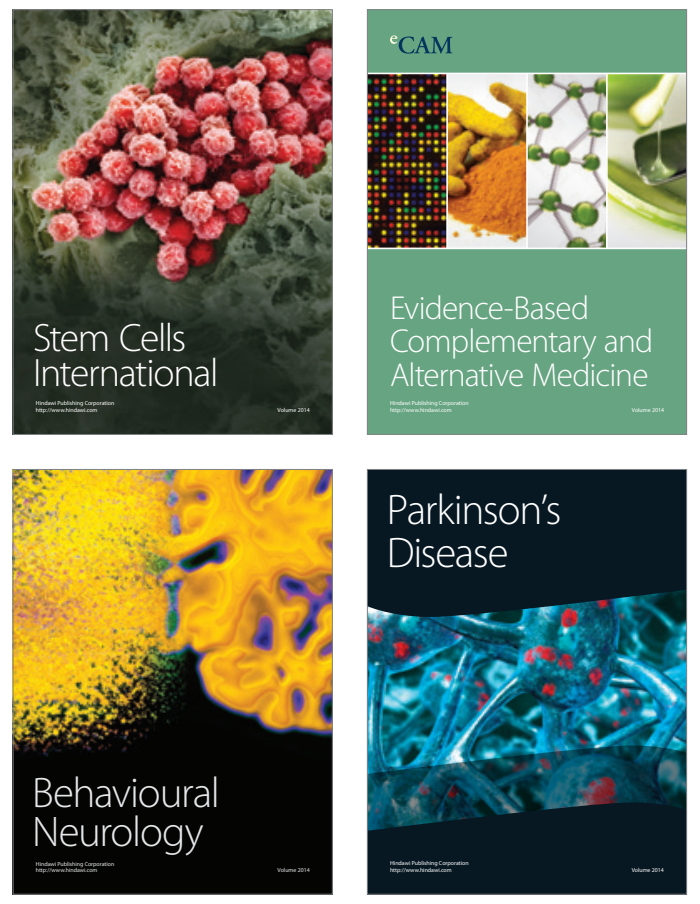
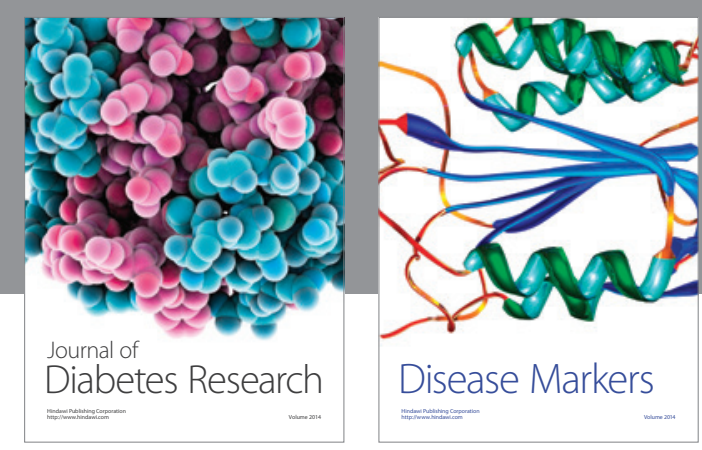

Disease Markers
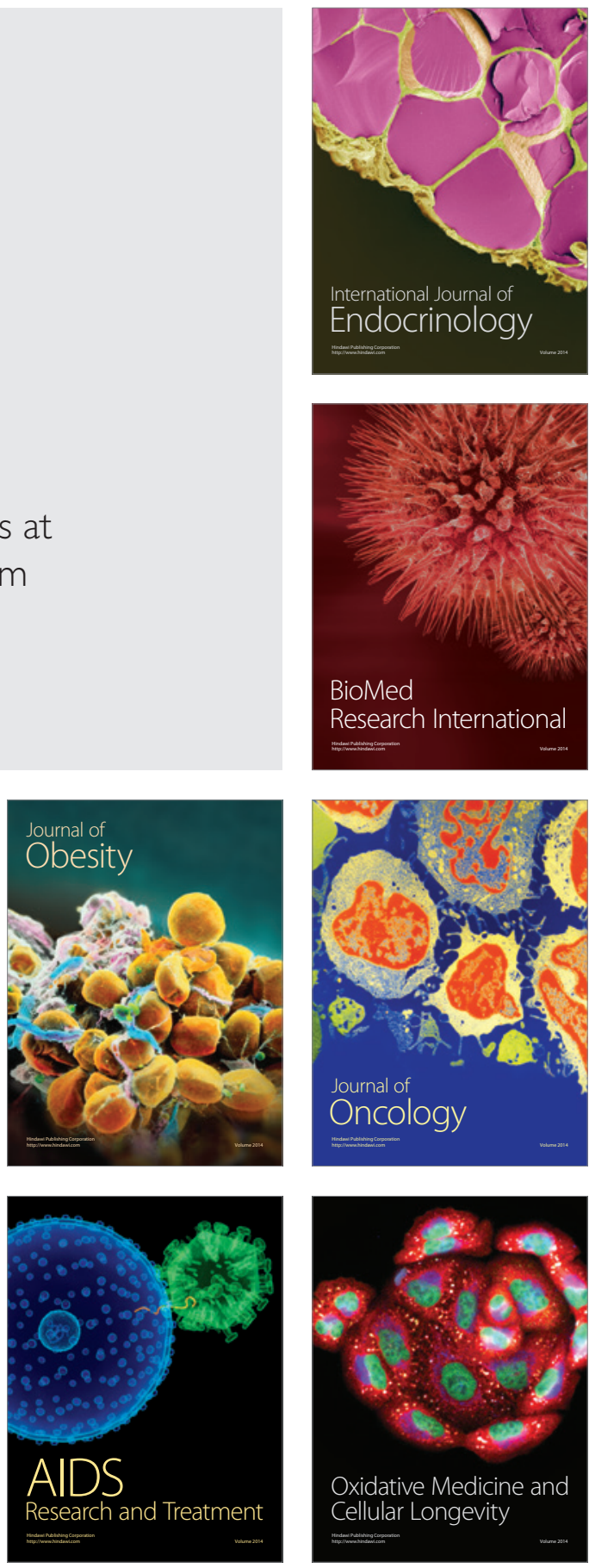